\title{
Nurses' documentation of falls prevention in a patient centred care plan in a medical ward
}

\section{AUTHORS}

CAGLAYAN YASAN Dip(Mgmt) MEd BSc Cert IV ${ }^{1}$ TRISH BURTON PhD MEd BSc BAppSc Dip(AppSc) RN FCNA ${ }^{2}$

MARK TRACEY MBiostat $\mathrm{BSc}^{3}$
1 College of Health and Biomedicine, Victoria University, Melbourne, Victoria, Australia.

2 Nursing and Midwifery, College of Healthcare Sciences, James Cook University. Queensland, Australia.

3 The Northern Hospital, Northern Health, Epping, Victoria, Australia.

\section{CORRESPONDING AUTHOR}

CAGLAYAN YASAN College of Health and Biomedicine, Victoria University, PO Box 14428, Melbourne, Victoria, Australia. 8001. Phone: + 61339912131

Email: Caglayan.Yasan@vu.edu.au

\section{ABSTRACT}

Objective: To evaluate the consistency of nurses' documentation in the falls prevention assessment tool, and to ascertain whether patients identified as high risk of falling had falls preventative strategies implemented.

Background: Falls are one of the leading causes of adverse events for patients in the hospital setting. The current practice of implementing falls prevention strategies for patients has not been able to be sustained, which remains a challenge for healthcare providers. Among the falls prevention strategies, falls risk assessment tools have been identified as a crucial element in falls prevention so as the number of falls are minimised.

Study design and methods: A retrospective chart audit, with the auditing of falls assessment documentation on the Patient Centred Care Plan.

Results: The Patient Centred Care Plan audit revealed that $60.8 \%$ of patients $(n=508)$ were identified as high risk of falls by the principal investigator. For the cohort of patients identified by the nurses as having a high risk of falling (53.4\%), $53.7 \%$ of patients had falls prevention strategies implemented, and only $17.5 \%$ of patients were engaged with their falls prevention plan. The strategies that were documented by the nurses on the care plan for the high-risk cohort were not implemented for $16.8 \%$ of the patients, and $29.5 \%$ of high risk of falls patients did not have documentation on the plan indicating their falls status.

Discussion: The findings show that there is a significant gap in the identification of high falls risk patients and the documentation and implementation of falls prevention strategies, between nursing staff records on the Patient Centred Care Plan and the audit conducted by the principal investigator for patients who are identified as high falls risk. As part of the audit patient engagement in their falls prevention plan revealed that patients were not informed of their falls risk status by the nursing staff.

Conclusion: The outcome from this audit signifies that not all high falls risk patients were identified as a high falls risk, and most of the high falls risk patients were not engaged in their falls prevention plan.

Implications for research, policy and practice: Understanding the current practices of falls prevention and raising nursing staff awareness of 
variance in the implementation of falls prevention strategies will improve the quality, efficiency of healthcare and patient safety.

Key words: Patient falls, assessment, implementation of strategies, patient engagement, health education

What is already known about the topic?

- Nurses' do not always document patient assessment and associated nursing care.

- There are multiple factors which impact on nurses' documenting assessment findings and implementation of nursing care.
What this paper adds:

- At times nurses rely on a falls risk assessment made on the previous day when not able to conduct a current falls assessment.

- Patients are not engaged in their falls prevention plan, even though they are identified as being of risk of falls.

- Patients are not aware of the implemented falls prevention strategies which are part of their care.

\section{INTRODUCTION}

Falls are multifactorial and there are several factors which may contribute to implementation of falls prevention strategy. These may include nursing staffs' knowledge, belief, attitudes, workloads and staff culture on the ward, environmental factors and access to required resources. ${ }^{1}$ Falls assessment tools have been documented to be effective in reducing falls rates and serious injuries amongst inpatients. ${ }^{1}$ Falls assessment tools help identify high falls risk patients, so that timely interventions can be put in place. ${ }^{2}$

The 6-PACK (STRATIFY) falls prevention tool was developed in the UK, ${ }^{3}$ and has been studied and implemented in Australian, Canadian and European acute care hospitals. ${ }^{4}$ The 6-PACK falls prevention tool is a nurse led assessment tool designed for acute care settings. As the tool is an accurate predictor of falls risk it has been recommended for use in best practice guidelines to prevent patient falls. ${ }^{4}$ ArandaGallardo and colleague's (2015) longitudinal study of the STRATIFY falls prevention tool, highlights that this tool maintains validity and accuracy as a predictor of falls risk. ${ }^{5}$

\section{PATIENT CENTRED CARE PLAN}

In 2002, the hospital in this study implemented the nurse led 6-PACK falls prevention tool, as part of the Patient Centred Care Plan (PCCP). The PCCP is a patient's daily plan of care which is completed by nursing staff and updated each shift and is located in the patient's medical record. The falls prevention strategies documented on the falls prevention tool are: the placement of falls alert signs above the patients' bed, use of high low beds, establishment of a toileting regime, supervision of patients whilst in the bathroom, use of bed/chair alarms, ensuring walking aid is within reach, and patient engagement/education in falls prevention plan. The implementation of the 'Alert Sign' with one or more falls prevention strategies specified in the PCCP are deemed as correct implementation of the falls prevention plan. As part of the implementation of the tool, the new nursing staff are provided training on their falls prevention risk assessment tool as part of the orientation to the hospital. Ward-based training was delivered by the injury prevention champions of the designated wards, so as to prepare and engage nursing staff in providing falls prevention assessment and interventions. The injury prevention champions of the ward conducted monthly audits of the falls prevention assessment tool, and in response to the audit results feedback was provided to the nursing staff team each shift. The nursing staff were encouraged to instil the education provided to the next shift to improve the assessment, documentation and implementation of the documented falls prevention strategies. Also, during ward handover/huddles the location of the high falls risk patients is reinforced by the nurse in charge to increase nursing staff awareness.

In 2002, as part of the introduction of the falls prevention tool in the hospital that is the focus of this study a nine-year observational evaluation study demonstrated $>80 \%$ compliance with falls prevention documentation. ${ }^{6}$ Barker and colleagues reported a $25 \%$ reduction in falls rates over an initial 12 month period and in the second year of implementation, a 50\% reduction. ${ }^{6}$ There continued to be a sustained decrease in the number of falls for five years post implementation with use of this tool in the hospital setting. ${ }^{4}$

The hospital has continued to use the falls prevention tool as part of its falls prevention program. Falls incidents were also classified according to an Incident Severity Rating (ISR): 'A score of $1,2,3$ or 4 that measures the severity of the impact caused to the person affected following an incident, ISR 1 being the highest or most severe and ISR 4 a near miss. ${ }^{7}$

Despite ongoing use of the tool, in the 2015 to 2016 period, there was a $16 \%$ increase (Table 1 ) in the overall number of the reported falls (ie. 147 to 170), consisting predominantly of an increase in no harm/near miss events (ISR-4) and with an increase in the number of serious injuries (ISR-2) in the medical ward. The PI abstracted the data from RiskMan, which is the hospital's information management system. In response to the increased incidence of patient falls in 2015-2016, an audit was instigated to determine the factors 
that have influenced this increase as the falls prevention tool has been a consistent component of the hospital's fall prevention strategy.

TABLE 1: FALLS INCIDENT SEVERITY RATING (ISR) 1 TO 4 AND THE TOTAL NUMBER OF FALLS FROM JAN 2015 TO DEC 2016

\begin{tabular}{|l|r|r|r|}
\hline Falls Severity Level 1-4 & \multicolumn{2}{|r|}{ Total Number of Falls } & Change \\
\cline { 2 - 4 } & $\begin{array}{r}\text { Jan-Dec } \\
2015\end{array}$ & $\begin{array}{r}\text { Jan-Dec } \\
2016\end{array}$ & $\%$ \\
\hline $\begin{array}{l}\text { Incident Severity Rating 1: } \\
\text { Severe (death) }\end{array}$ & 1 & 1 & 0 \\
\hline $\begin{array}{l}\text { Incident Severity Rating 2: } \\
\text { Moderate (head injury, } \\
\text { subdural haematoma, fracture) }\end{array}$ & 2 & 5 & $\uparrow 150$ \\
\hline $\begin{array}{l}\text { Incident Severity Rating 3: } \\
\text { Mild (graze, abrasion, cuts) }\end{array}$ & 47 & 46 & $\downarrow 2$ \\
\hline $\begin{array}{l}\text { Incident Severity Rating 4: } \\
\text { No harm/near miss }\end{array}$ & 97 & 118 & $\uparrow 21$ \\
\hline $\begin{array}{l}\text { Total number of falls } \\
\text { 2015-2016 }\end{array}$ & 147 & 170 & $\uparrow 16$ \\
\hline
\end{tabular}

The aim of the present study was to determine the number of patients admitted to the medical ward identified by nurses as high falls risk, measure the consistency of nurses' documentation of the identified falls prevention strategies using the falls prevention tool, and assess whether preventative falls prevention strategies were implemented for these patients.

\section{METHODS AND METHODOLOGY}

Quantitative data collection consisted of RiskMan falls data and PCCP Audit. RiskMan is an information management system used by hospitals to report in-house incidents. The data obtained from RiskMan is the basis for the instigation of quality improvement projects at the hospital. The PCCP Audit facilitated a comparison between the principal investigator (PI) and nursing staff identifying the patients as high risk of falls and the data was aggregated statistically. ${ }^{8}$ RiskMan data results are presented as total number of falls and percentages. The results demonstrated different patterns in the identification, assessment and documentation of implemented falls prevention strategies. ${ }^{9}$

\section{ETHICAL CONSIDERATIONS}

The audit is part of the Doctor of Philosophy research study, and the rights of patients and nursing staff were upheld. The data (patient medical records and RiskMan data) was initially collected in an identifiable format but once the datasets had been linked, the data was de-identified. Verbal and written consent was obtained from nursing staff. The approval for the research study was given by the Ethics Committee of Austin Hospital HREC Project Number: HREC17 Austin27 and Victoria University Ethics Committee.

\section{PARTICIPANTS}

The principal investigator (PI) is a registered nurse and worked as an Injury Prevention Coordinator in the hospital for two years where the study was conducted. The Injury Prevention Coordinator role was responsible for the continuing education of nursing staff in providing safe patient focussed nursing care. Part of this continuing education involved the documentation and implementation of the falls prevention risk assessment tool. The nurses were registered nurses who provided nursing care to the patients in the medical ward. The ward staff consisted of graduate nursing staff, registered nurses, clinical nurse specialist, associate nurse unit manager and the nurse unit manager. Nurses on the ward use a team approach when providing patient care and there is a strong culture for collaboration. After the monthly auditing of the falls prevention assessment tool, targeted education sessions were conducted by the injury prevention champions of the ward.

\section{AUDIT SITE}

A point prevalence audit of the PCCP was conducted by the PI from 20 July 2017 to 30 November 2017 in the 28-bed medical ward at a major hospital in Melbourne. The ward has the allocation of 16 dementia and four renal dialysis beds. The audit was conducted each Wednesday for twenty weeks of the PCCP.

\section{DATA COLLECTION}

The audit was undertaken in the afternoon by the PI so as the nursing staff on the morning shift has adequate time to complete the falls prevention risk assessment tool. Given that the same patient could be audited more than once, the audit results are presented in the unit of patient beds. The PI audited the falls prevention risk assessment tool documentation by the nursing staff, and also used the same falls prevention risk assessment tool to rate the patient's falls risk. The PCCP audit results provided information on the nursing assessment of the patient, documentation and implementation of targeted strategies to prevent falls on the medical ward. Furthermore, the audit provided a comparison between the nursing staff and PI identifying the patients falls risk score and patient engagement in their falls prevention plan. As per the hospital's falls prevention policy, identified high falls risk patients are required to be engaged in their falls prevention management plan by nursing staff, as falls prevention is part of a high falls risk patient's daily management plan. The nursing staff are required to discuss the patient's falls risk, implement falls prevention strategies and highlight the falls prevention patient brochure with the patient and clarify/answer any questions a patient or significant other may have in regard to their falls prevention plan. Once the education session is completed the date and who the education was provided by is documented on the PCCP to indicate to other nursing staff that falls education was provided. 


\section{DATA ANALYSIS}

Descriptive analysis was conducted to provide an indication of the falls risk assessment tool completion and the falls risk minimisation methods utilised and in practice for each patient. Chi-squared test and Fishers' exact tests were used to test for differences between the registered nurses and the principal investigator, while continuous variables were assessed for normality, with the appropriate parametric (Student's t-test) and/or non-parametric (Man-Whitney) tests applied. All analyses were conducted using STATA statistical analyses software, version 15.1 (StataCorp, College Station, TX, USA), with a two-sided p-value of less than 0.05 indicating statistical significance. The number of statistical tests have been kept to a minimum to reduce the likelihood of false positive results, and to avoid any adjustment for multiple comparisons, such as a Bonferroni adjustment. ${ }^{14}$

\section{RESULTS}

From a review of nurse-documented falls assessment as indicated in Table 2, the documentation compliance of entering a RiskMan number on the PCCP was only $60 \%(n=18)$.

Of the 508 patient beds audited, 271 were identified as a high falls risk by nursing staff with an additional 38 patients identified as high falls risk by the PI ( $p=0.016)$. Sixty-one percent of the patients admitted to the medical ward over the audit period were identified as having a high risk of falls. The differences between the nursing staff and PI's assessment of patients being a high risk of falls was statistically significant ( $p=0.016$ ). Of the $60.8 \%$ of high-risk falls patients, only $53.7 \%$ had falls preventative strategies implemented as part of their nursing care (Classification of strategies implemented: Alert sign and one or more strategies implemented is deemed accurate). Seventeen percent of strategies documented on the PCCP were not implemented and $29.5 \%$ of high risk of falls patients had no documentation on PCCP as indicated in Table 2.

As shown in Table 2, of the 309 patients identified as high falls risk by the PI, only $17.5 \%$ were provided with Falls Prevention Brochures, which indicates that $83 \%$ of patients were not engaged in their Falls Prevention Plan. Out of 309 patients identified as high falls risk, 2.6\% were confused, and 2.6\% were non-English-speaking background.
TABLE 2: PCCP AUDIT RESULTS OF RISKMAN NUMBER DOCUMENTATION, IDENTIFICATION OF PATIENTS' FALLS RISK SCORE AND ENGAGEMENT

\begin{tabular}{|c|c|c|c|}
\hline Criteria/Questions & Number & $\begin{array}{r}\text { Total } \\
\text { Occupied } \\
\text { Beds }\end{array}$ & $\%$ \\
\hline Falls this admission & 30 & 508 & $5.9 \%$ \\
\hline $\begin{array}{l}\text { RiskMan No. documented on } \\
\text { PCCP }\end{array}$ & 18 & 30 & $60 \%$ \\
\hline $\begin{array}{l}\text { Staff identified patient as } \\
\text { high risk of falls }\end{array}$ & 271 & 508 & $53.4 \%$ \\
\hline $\begin{array}{l}\text { PI identified patient as high } \\
\text { risk of falls }\end{array}$ & 309 & 508 & $60.8 \%$ \\
\hline $\begin{array}{l}\text { Falls preventions strategies } \\
\text { implemented }\end{array}$ & 166 & 309 & $53.7 \%$ \\
\hline $\begin{array}{l}\text { Falls prevention not } \\
\text { documented in PCCP }\end{array}$ & 91 & & $29.5 \%$ \\
\hline $\begin{array}{l}\text { Falls prevention not } \\
\text { implemented }\end{array}$ & 52 & & $16.8 \%$ \\
\hline $\begin{array}{l}\text { Falls prevention brochure } \\
\text { provided }\end{array}$ & 54 & 309 & $17.5 \%$ \\
\hline $\begin{array}{l}\text { Falls prevention brochure not } \\
\text { provided }\end{array}$ & 255 & & $82.5 \%$ \\
\hline $\begin{array}{l}\text { Risk and strategies discussed } \\
\text { with patient }\end{array}$ & 14 & & $25.9 \%$ \\
\hline $\begin{array}{l}\text { Risk and strategies not } \\
\text { discussed with patient }\end{array}$ & 40 & & $74.1 \%$ \\
\hline Patient confused & 8 & & $2.6 \%$ \\
\hline Patient NESB & 8 & & $2.6 \%$ \\
\hline
\end{tabular}

Table 3 provides an indication of which falls prevention strategies were implemented and not implemented. Of the 309 patients with falls risk $58 \%$ of patients had 'Alert Signs' as a falls risk displayed above their beds, whilst $28 \%$ of patients did not have falls risk documentation in their PCCP. In relation to the intervention of a hi-low bed as part of a risk of falls patient's care, $71 \%$ of patients did not have the intervention of a 'high low bed' documented or implemented. Of the $86 \%$ of high falls risk patients requiring a 'gait aid', only $25 \%$ of these patients had their walking aids within reach. Only $2 \%$ of 'toileting regimes' and $43 \%$ of high falls risk patient's 'bathroom supervision' were documented as implemented on the PCCP. For the 14 patient beds with an alarm in-situ, $64 \%$ of the patient 'alarms' were not connected correctly. 
TABLE 3: IMPLEMENTED/NOT IMPLEMENTED 6-PACK FALLS PREVENTION STRATEGIES IDENTIFIED ON THE PCCP

\begin{tabular}{|c|c|c|c|}
\hline Alert sign & Number & $\begin{array}{r}\text { Total No. of } \\
\text { High-Risk } \\
\text { Patients }\end{array}$ & $\%$ \\
\hline $\begin{array}{l}\text { Alert sign documented on } \\
\text { PCCP as implemented }\end{array}$ & 178 & 309 & $58 \%$ \\
\hline Alert sign not implemented & 43 & & $14 \%$ \\
\hline No documentation in PCCP & 88 & & $28 \%$ \\
\hline \multicolumn{4}{|l|}{ Low bed } \\
\hline $\begin{array}{l}\text { Low bed documented on PCCP } \\
\text { as implemented }\end{array}$ & 90 & 309 & $29 \%$ \\
\hline If so, is it in lowest position & 68 & & $76 \%$ \\
\hline $\begin{array}{l}\text { No documentation and no } \\
\text { implementation on PCCP }\end{array}$ & 219 & & $71 \%$ \\
\hline \multicolumn{4}{|l|}{ Gait aid } \\
\hline $\begin{array}{l}\text { Total no. of high risk patient } \\
\text { require gait aid }\end{array}$ & 267 & 309 & $86 \%$ \\
\hline Gait aid provided & 63 & 267 & $24 \%$ \\
\hline If so, is it close to patient & 43 & 63 & $68 \%$ \\
\hline \multicolumn{4}{|l|}{ Toileting regime } \\
\hline $\begin{array}{l}\text { Toileting regime documented } \\
\text { on PCCP as commenced }\end{array}$ & 5 & 309 & $2 \%$ \\
\hline $\begin{array}{l}\text { Toileting regime not } \\
\text { commenced }\end{array}$ & 210 & & $68 \%$ \\
\hline No documentation on PCCP & 94 & & $30 \%$ \\
\hline \multicolumn{4}{|l|}{ Bathroom } \\
\hline $\begin{array}{l}\text { Supervise bathroom } \\
\text { documented }\end{array}$ & 133 & 309 & $43 \%$ \\
\hline $\begin{array}{l}\text { Supervise bathroom not } \\
\text { documented }\end{array}$ & 114 & & $37 \%$ \\
\hline No documentation in PCCP & 59 & & $19 \%$ \\
\hline \multicolumn{4}{|l|}{ Alarm insitu } \\
\hline $\begin{array}{l}\text { Total no. of high-risk patient } \\
\text { requires alarm }\end{array}$ & 128 & 309 & $41 \%$ \\
\hline Alarm insitu & 14 & 128 & $11 \%$ \\
\hline If so, is it connected correctly & 5 & & $36 \%$ \\
\hline $\begin{array}{l}\text { Documented PCCP but not } \\
\text { implemented }\end{array}$ & 9 & & $64 \%$ \\
\hline
\end{tabular}

\section{DISCUSSION}

This study identified variability between patients being identified as high falls risk when comparing nursing staff records on PCCP and the audit conducted by the PI.

Beauchet and colleagues similarly found that $13.5 \%$ of patients that were audited to be a high risk of falls were not identified as risk of falling. ${ }^{10}$ Accurate documentation and the reporting of falls is crucial in improving the quality of patient care. The findings suggest that there is a significant gap in the identification of high falls risk patients and the documentation and implementation of falls prevention strategies, along with patient engagement in their falls prevention plan. Knowing that many falls occur in bathrooms due to toileting, which could potentially result in serious injuries, ${ }^{2}$ the barriers of not documenting high falls risk patient's toileting regime in the PCCP needs to be identified to improve patient outcomes. Some of the barriers that impact upon nurses' adherence to documentation may have been due to demands of patient care requirements (dementia, confused, delirium, and renal impairment). Also, the patient requirements may not always correlate with staff capacity due to workloads, complex patient care needs, staff allocation and skill mix.

Not displaying the 'alert signs' for high falls risk patients above their beds will hinder strategies to reduce falls rates. The allocation of alert signs increases the awareness of a patient's risk of falling to everyone who encounters the patient's surroundings, including the multidisciplinary team of nurses, doctors and visitors, and even the tea person. Radecki and Reynolds (2018) found that it was important that the patient was also aware of their falls risk, so that the patient could be an active participant in the falls prevention interventions. ${ }^{11}$ It is imperative to document that a patient falls prevention brochure is provided to ensure that patients are engaged in their falls prevention plan.

Ensuring a patient's walking aid is within their reach allows the patient to stabilise their posture/mobility which in turn minimises their risk of falling. The use of bed and chair alarms for cognitively impaired patients play a crucial role in the minimisation of falls, as the alarms alerts healthcare professionals of movement when a patient is attempting to ambulate without any supervision. From the patient perspective, Radecki and Reynold found that patients' considered alarms to be part of the falls risk strategy. ${ }^{11}$ In a medical ward where 16 dementia specific beds are located, having alarms not connected correctly can result in a serious injury due to a fall. Upon investigation for the reasons why there were a high number of alarms not documented or connected correctly, it was highlighted by nursing staff that the documentation was copied from the day before.

Patient centred care or patient engagement is a fundamental care requirement of the healthcare system, and by involving the patient in their daily care plan, evidence suggests that this will decrease the number of falls. ${ }^{11}$ As per National Safety and Quality Health Service Standards [NSQHS] criteria 10.9.1 patients and their carers are required to be informed of their identified falls risk and engaged in their falls prevention strategies and management plan. ${ }^{2}$ Patients' cognitive impairment or language barriers at times can hinder the patient education process. ${ }^{13}$ As per the hospital's policy the nursing staff are required to provide a falls prevention brochure and discuss the strategies implemented with the high falls risk patient. The falls prevention brochures are translated into multiple languages and available for nursing staff to print and provided to the non-English speaking 
patients. In a medical ward by not actively engaging high falls risk patients in their falls prevention plan, not only increases their risk of falling, but also, prevents patient awareness of their falls risk to undertake the targeted strategies implemented/required whilst in hospital or post discharge. ${ }^{11}$

Knowing that the 6-PACK interventions are utilised as part of patients' daily care to decrease the number of serious injuries, it is important to obtain nurses perceptions to understand what constitutes the implementation of targeted falls prevention strategies on the falls prevention tool. The results of this study were provided to nursing staff/nurse unit manager of the ward during focus group discussions and the hospital's Standard 1o falls prevention committee to improve local nursing practice.

\section{CONCLUSION}

Even though the falls risk assessment tool was feasible to implement and has previously reduced the fall and fall related serious injury rate at the current hospital, the falls rate and reported serious injuries appears to be a continuing problem. A possible explanation for serious injuries on the rise is the inconsistent implementation of the falls prevention strategies. This study indicates that the nurse's risk assessment and implementation of falls prevention strategies as documented in the falls assessment tool, was not being applied as it is intended, and may not be having the same effect of reducing the rate of falls as it did following its introduction to the hospital in 2002. It is imperative to identify the barriers and the enablers to further understand the reasons behind nurses' documentation and the apparent lack of implementation of the targeted strategies. While the above audit results provide an insight, further research is required to explore the nurses' and patients' perspectives on effectiveness of the current falls prevention plan is required. The next phase of this study will explore these views.

\section{RECOMMENDATIONS}

An analysis should be conducted to determine the long-term sustainability of the falls prevention program. As part of the analysis the identification of the barriers and enablers that impact upon nurses' documentation and implementation of targeted falls strategies, requires further examination. The further exploration of both nursing staff and patients' perspectives regarding the effectiveness of current falls prevention plan, will contribute the overall analysis.

Funding support: No funding was received for this project.

Declaration of conflicting interests: No conflict of interest has been declared by the authors.

Authors' contribution: Caglayan Yasan: study design, manuscript preparation, data collection and analysis. Mark Tracey: analysis and interpretation of statistical data. Trish Burton: manuscript preparation, critically reviewed and approved the manuscript prior to submission.

\section{REFERENCES}

1. Barker AL, Morello RT, Ayton RA, Hill KD, Landgren F S, Brand CA. Development of an implementation plan for the 6-PACK falls prevention programme as part of a randomised controlled trial: protocol for a series of preimplementation studies. Inj Prev. 2015; 22(2):1-7. DOI:10.1136/injuryprev2015-041915

2. Morello RT, Barker AL, Ayton DR, Landgren F, Kamar J, Hill KD, Brand CA, Sherrington C, Wolfe R, Rifat S, Stoelwinder J. Implementation fidelity of a nurse-led falls prevention program in acute hospitals during the 6-PACK trial. BMC Health Serv Res. 2017; 17:383. DOI:10.1186/s12913-0172315-z

3. Oliver D, Britton M, Martin FC, Hopper AH. Development and evaluation of evidence-based risk assessment tool (STRATIFY) to predict which elderly inpatients will fall: case-control and cohort studies. BMJ. 1997; 315(7115): 1049-53. DOI:10.1136/ bmj.315.7115.1049

4. Barker A, Kamar J, Graco M, Lawlor V, Hill K. Adding value to the STRATIFY falls risk assessment in acute hospitals. J Adv Nurs. 2011; 67(2):450-7. DOI:10.1111/j.1365-2648.2010.05503.x

5. Aranda-Gallardo M, Enriquez de Luna-Rodriguez M, CanceSanchez JC, Moya-Suarez AB, Morales-Asencio JM. Validation of the STRATIFY falls risk assessment tool for acute-care hospital patients and nursing home residents: study protocol. J Adv Nurs. 2015; 71(8):1948-57. DOI:10.1111/jan.12651

6. Barker A, Brand C, Haines T, Hill K, Brauer S, Jolley D, Botti M, Cumming R, Livingston PM, Sherrington C, Zavarsek Silva, Morello R, Kamar J. The 6 PACK programme to decrease fall-related injuries in acute hospital: protocol for a cluster randomised controlled trial. Inj Prev. 2011; 17:e5. DOI:10.113/ injuryprev-2011-040074.

7. Department of Health. Victorian Health Incident Management Policy. 2011; 27 Retrieved from: https://www2.health.vic.gov.au/ hospitals-and-health services/quality-safety-service/clinicalrisk-management

8. Patton MQ. Qualitative Research \& Evaluation Methods. $3^{\text {rd }}$ ed. Sage. United States. 2002

9. Hays DG, Singh AA. Qualitative inquiry in clinical and educational settings. Guilford Press. New York. 2012

10. Beauchet $O$, Noublanche F, Simon R, Sekhon H, Chabot J, Levinoff EJ, Kabeshova A, Launay CP. Falls Risk Prediction for Older Inpatients in Acute Care Medical Wards: Is there an interest to combine an early nurse assessment and the artificial neural network analysis? J Nutr, Health Aging. 2018;(22)1:131-7. DOl:10.1007/s12603-017-0950-z

11. Radecki B, Reynolds S, Kara A. Inpatient fall prevention from the patient's perspective: A qualitative study. Appl Nurs Res. 2018; 43:114-9. DOI:10.1016/j.apnr.2018.08001.

12. Australian Commission on Safety and Quality in Health Care. Standard 10 Preventing Falls and Harm from Falls Safety and Quality Improvement Guide. Criterion: Communicating with patients and carers. Sydney. 2012. Retrieved from: https:// www.safetyandquality.gov.au/sites/default/files/migrated/ Standard10 Oct 2012 WEB.pdf

13. Sonnad SS, Mascioli S, Cunningham J, Goldsack J. Do patients accurately perceive their falls risk? Nursing. 2014; 44(11):58-62 DOI:10.1097/01.NURSE.0000454966.87256.f7.

14. Schulz KF, Grimes DA. Multiplicity in randomised trials I: endpoints and treatments. Lancet. 2005; 365(9470):1591-5. 